\title{
Chapter 2 \\ Influence of Binocular Disparity in Depth Perception Mechanisms in Virtual Environments
}

\author{
Matthieu Poyade, Arcadio Reyes-Lecuona, and Raquel Viciana-Abad
}

\begin{abstract}
In this chapter, an experimental study is presented for evaluating the importance of binocular disparity in depth perception within a Virtual Environment (VE), which is assumed to be critical in many manipulation tasks. In this research work, two assumptions are made: Size cues strongly contaminate depth perception mechanisms and binocular disparity optimizes depth perception for manipulation tasks in VE. The results outline size cues as possible cause of depth perception degradation and binocular disparity as an important factor in depth perception, whose influence is altered by the position within a VE.
\end{abstract}

\subsection{Introduction}

In certain industrial environments, the simulation of design and training processes is necessary and often involves manipulation tasks. Nevertheless, depending on the nature of the industrial task, the simulation of these processes under real world conditions may result limited and expensive due to logistical needs. In contrast, manipulation in Virtual Environments (VE) may become an interesting alternative to simulators based in real environments. Nowadays, technological improvements concerning virtual reality devices allow for the design of reliable simulations, easily reconfigurable and with a reasonable cost.

In the specific case of manipulation in VEs, the perception of depth is a decisive factor. The goal of this work is to provide a better understanding of depth perception mechanisms. The experimental study performed consists of two stages. First, the study evaluated the magnitude of sensorial conflicts generated by objects size in depth perception within a VE. Then, the study focused on the influence of binocular disparity in the process of depth perception.

\footnotetext{
M. Poyade ( $\otimes)$

Departamentos de Technología Electrónica, ETSI de Telecomunicación Universidad de Málaga, Spain

e-mail: matthieu.poyade@uma.es
} 


\subsection{Prior Work}

Many studies have demonstrated the advantages of using stereoscopic visualization in VEs [1-4]. Stereoscopic cues provide a noteworthy improvement of depth perception in a very realistic way $[5,6]$, intensifying perception of surfaces and materials [7], and also facilitating spatial localization and navigation. Therefore, the already proved benefits of stereoscopic visualization support its implementation within VEs in which an accurate manipulation is required [3].

\subsubsection{Depth Perception Mechanisms in Real World and Depth Cues Theory}

Human visual system consists of very complex mechanisms, which are able to perform complex tasks, such as simultaneously processing two visual stimuli received from both eyes and generating a three-dimensional mental model. This mechanism is well known as stereopsis and it refers to the visual system capacity of computing coherently two monocular signals to create a three-dimensional view of an environment.

According to the literature, stereoscopic view depends on binocular and oculomotor depth cues [7]. Binocular depth cues refer to the depth sensation provided by the stereopsis by means of processing the slightly different retinal images of both eyes, resulting from the human eyes horizontal separation. It is commonly assumed that human eyes separation known as the average interocular distance, ranges from $6.3 \mathrm{~cm}$ to $6.5 \mathrm{~cm}$ [8]. Oculomotor depth cues comprise the sight accommodation and convergence processes [7].

Depth cues theory refers to depth provided not only by binocular and oculomotor depth cues but also by monocular depth cues. These last cues are known as pictorial cues and are related to depth information provided by images. The most common pictorial cues are occlusion, size, shade, illumination, texture, and color [6, 7].

The processing of depth cues by the visual system provides the sensation of depth related to an object and its surrounding. Many works [6, 9-11] suggested the study of depth perception taking into account both pictorial and stereoscopic (binocular and oculomotor) depth cues.

In a stereoscopically displayed VE, the control of the stereoscopic cues is essential in order to avoid sensorial conflicts due to accumulation of various cues.

\subsubsection{Stereopsis and Depth Perception in a Virtual Environment}

Depth Perception in Computer Generated Images (CGI) is strongly enhanced by stereoscopic vision. Furthermore, depth perception has demonstrated to improve when depth information is provided by various depth cues associated to stereopsis 
instead of only pictorial depth cues [7]. Therefore, the VE must provide the appropriate depth cues.

Pfautz suggested a stereoscopic visualization omitting eyes accommodation. Convergence is naturally performed at the focal distance of the observed virtual scene. Therefore, only binocular depth cues supply depth information.

Binocular depth cues ensure that each eye has its own viewpoint of the CGI displayed on the screen. The display system supplies the stereopsis by displaying twice each CGI considering a horizontal shift corresponding to the natural interocular distance. Perceived depth is illustrated in Fig. 2.1.

Fig. 2.1 Perceived depth in front of and behind the display panel.

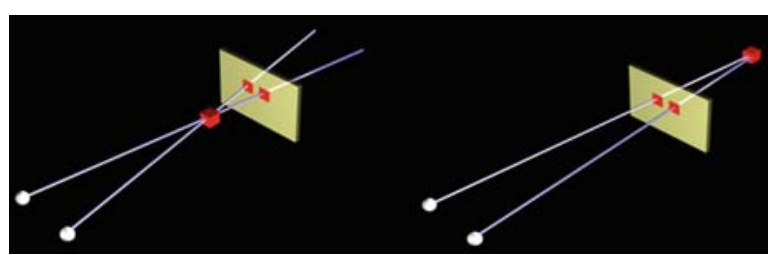

This figure will be printed in $\mathbf{b} / \mathbf{w}$

Equations 1 and 2 respectively show the inner and outer perceived $p$ depth from the projection area [12] as a function of the natural interocular distance $e$, the observation distance $z$ and the displayed binocular disparity onto the screen $d$ generated by the shifted CGI.

$$
\begin{aligned}
& p=z /((e / d)+1) \\
& p=z /((e / d)-1)
\end{aligned}
$$

\subsubsection{Stereoscopic Display Technical Principles}

OpenGL libraries are very efficient in generating stereoscopic visualization in VE. These libraries allow for the use of several display functions [13, 14], such as the orthogonal projection function in symmetrical or asymmetrical perspective known as frustum which specifies the parameters of the camera attached to the user viewpoints in the VE. Both eyes are represented by two cameras horizontally shifted by the interocular distance.

Jones [12] and Holliman [15] proposed a method to convert depth between VE and real world depending on the parameters of the OpenGL display function.

Many works have focused on the study of the depth perception in VEs. Nevertheless, depth perception is still not well understood and is controlled with difficulty due to the numerous interactions between pictorial and stereoscopic cues.

Rosenberg [4] has experimentally studied the extent to which the interocular distance influences depth perception. He demonstrated the degradation of depth perception to be a function of interocular distance when this distance exceeds certain 
limitations of a broad range. Nevertheless, his experimental design consisted in a depth comparison of identical objects located close to the zero parallax plane. His results were obtained attending to depth information provided by binocular cues, but objects' size might have strongly affected this perception.

One of the main goals of this experimental study is the evaluation of the influence of certain pictorial depth cues regarding the interocular distance in depth perception. This experimental study is based on two hypotheses. The first hypothesis establishes that object size influences depth perception in a higher extent than stereoscopic depth cues, while manipulating objects of identical shape. The second hypothesis considers the existence of an optimal binocular disparity to accurately perceive depth in a VE.

\subsection{Method}

Fifteen volunteers (10 males, 5 females) were recruited from among students from the School of Telecommunication Engineering of the University of Malaga. Participants were aged from 22 to $27(\mu=24, \sigma=1.69)$ and did not present any relevant visual deficiency. None of them were economically granted for his/her participation in this experiment.

The VE was implemented in Microsoft Visual C++ version 6.0 using the OpenGL libraries. Stereoscopic visualization was provided in the VE by using two asymmetrical oblique frustum functions shifted one from the other by the binocular distance, which was modified depending on the experimental conditions.

The experiment was performed on a PC (Pentium3 at $1 \mathrm{GHz}, 1 \mathrm{~GB}$ RAM and graphic card INTENSE3D Wildcat Pro $4210120 \mathrm{~Hz}$ ) with a flat CRT screen IIyama Visión Master Pro 514 (width $300 \mathrm{~mm}$, height $400 \mathrm{~mm}$ ) customized for stereoscopic visualization using a CrystalEYES ${ }^{\circledR}$ workstation from StereoGraphics ${ }^{\circledR}$ corporation. The workstation consisted of a pair of standard LCD shutter glasses and an infrared emitter. CrystalEYES ${ }^{\circledR}$ shutter glasses provided to each eye its respective viewpoint of the VE displayed on the screen while the infrared emitter synchronized each viewpoint frequency to the half of the graphic card rendering frequency, i.e. at 60 frames per second.

\subsubsection{Procedure}

Participants were seated in front of the projection area at an approximate distance of $1 \mathrm{~m}$. Their movements were restricted to the front of the display area. Interaction with the VE was based on virtual objects displacement using the keyboard. The screen was elevated in order to horizontally align participants' glance with the displayed virtual scene, as shown in Fig. 2.2a. 


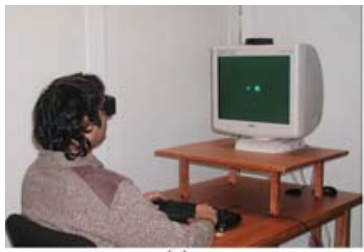

(a)

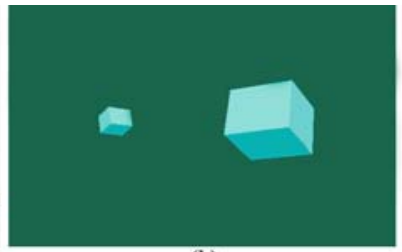

(b)

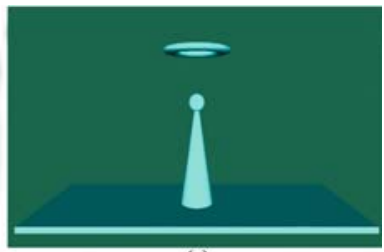

(c)

Fig. 2.2 (a) Installation of the experiment, (b) experimental design of the first stage, (c) experimental design of the second stage

Experimental design consisted of 15 scenarios which gathered different experimental conditions, according to a full factorial design, randomly ordered with an incomplete counter balanced design.

Before starting the experiment, participants performed a training trial in which they were asked to play with the virtual scene, by moving the virtual object inside and outside the screen. The objective was to detect whether they were affected by any visual disorder and also to get them familiarized with the stereoscopic display.

Then, participants received instructions concerning the aim of the experiment, design consideration (virtual objects may have different sizes) and temporal characteristics (duration: 35 minutes, 2 breaks strategically planned). Furthermore, they were informed about their right to rest whenever they wanted.

To evaluate participants' depth perception, the experiment consisted of different scenarios where they were asked to place an object at the same depth than a static reference. These scenarios were classified in two stages.

In the first experimental stage, the virtual scene consisted of two floating cubes colored and placed in a dark green background. The participants had to place the dynamic cube, located at their right side, at the same depth than the reference left object (see Fig. 2.2b). Movement of the cube was allowed in the $Z$-axis direction, inside and outside the screen, using the arrows keypad.

During the two sessions of this stage, depth perception was evaluated as a function of three factors (independent variables): the binocular disparity $(0.0 \mathrm{~cm}$, $1.0 \mathrm{~cm}, 3.0 \mathrm{~cm}, 6.4 \mathrm{~cm}$, and $9.0 \mathrm{~cm}$ ); the positions within the VE (inside the screen: $-17.9 \mathrm{~cm}$ of perceived depth, at parallax zero: $0.0 \mathrm{~cm}$ of perceived depth, and outside the screen: $18.0 \mathrm{~cm}$ of perceived depth) and the objects' size (both objects sized equal, one object was twice bigger than the other one and vice versa).

In the second experimental stage, the graphical design was made in order to avoid any influence of objects size. Therefore, an important contaminant factor of depth perception was cancelled. In this stage, the task consisted in positioning two different objects at equal depth. Participants had to place a horizontally laid torus onto a cone-shaped object, in such a way that if the torus was released, it would fit perfectly onto the base of the cone-shaped object (see Fig. 2.2c). Participants could only move the torus following the $Z$-axis direction, inside and outside the screen, by pressing the arrows keypad. Again, participants' errors during the placement in depth of the torus were used to evaluate their depth perception. As in the first stage, 
position and binocular disparity were controlled as independent variables. In both stages, the dependent variable was the difference of depth between the manipulated object and the reference object (left side cube or cone). The computation of this difference was made when participants confirmed the object position by pressing the space bar. These differences were related to distances in the virtual world expressed in Virtual Units (VU).

\subsection{Results}

The experimental results were analyzed with a two way ANOVA (ANalysis Of VAriance) of repeated measures. Furthermore, this analysis was carried out considering three independent factors (object size, position, and binocular disparity) in the first stage of the experiment and two (position and binocular disparity) in the second stage. In both stages, the dependent variable was the difference of depth position between both objects within the VE, referred onwards as error measure.

\subsubsection{Results of the First Stage}

The error measure was strongly and significantly influenced by the size factor $\left(F_{(2,13)}=28.789, p<0.001\right)$. As it was expected, when objects sized differently, error magnitude in the task of placement increased. Nevertheless, the influence of the binocular disparity factor $\left(F_{(4,11)}=1.196, p<0.366\right)$ and the position factor $\left(F_{(2,13)}=0.792, p<0.474\right)$ in the error measure were not found significant.

This analysis also revealed the existence of a significant interaction between size and position factors $\left(F_{(4,11)}=7.734, p<0.003\right)$. Furthermore, the interaction between size and binocular disparity factors was also nearly significant.

Figure 2.3 shows the magnitude of the average error in the task of placing the virtual object in depth as a function of the three previously specified factors. As it can be seen in this figure, the difference in the depth position estimated between the dynamic object and the reference one was influenced by the difference in their sizes. Thus, the placement errors were of high magnitude and with a positive sign when the dynamic object was the biggest, while these errors were also of high magnitude but negatively signed when dynamic object was the smallest. So the participants unconsciously relied on the size cues to place dynamic objects. Moreover, the influence of the binocular disparity on the magnitude of the average error can also be seen in Fig. 2.3. Thus, the more the binocular disparity increased, the more the magnitude of the average error decreased. As well, the average error magnitude was more important in the case of further objects (Fig. 2.3a) than in the case of closer objects (Fig. 2.3.c). 


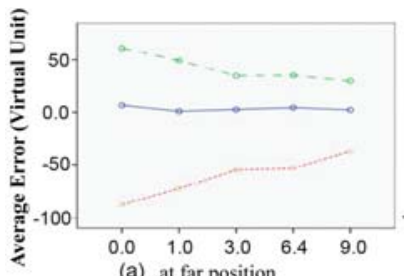

(a) at far position

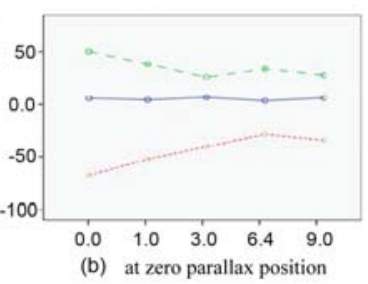

Object size relation

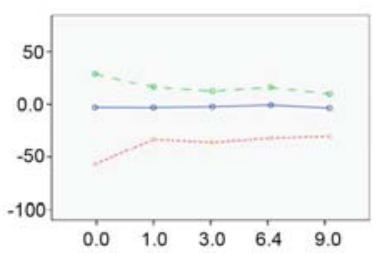

(c) at near position

\begin{tabular}{|l|}
\hline This \\
figure \\
will be \\
printed \\
in b/w
\end{tabular}

Fig. 2.3 Results of the first stage of the experiment. Curves representing the average error evolution in the task of placement in depth as a function of binocular disparity (expressed in $\mathrm{cm}$ ) with different size relation

\subsubsection{Results of the Second Stage}

The analysis of the second stage revealed that both cues significantly influenced depth perception: position $\left(F_{(2,13)}=10.853, p<0.002\right)$ and binocular disparity $\left(F_{(4,11)}=9.710, p<0.001\right)$. Results also showed a significant interaction between both factors $\left(F_{(7,8)}=7.567, p<0.007\right)$.

The influence of both factors in the error measure is shown in Fig. 2.4. Each curve shows the results for one of the three possible positions within the VE.

Fig. 2.4 Results of the second stage. Curves representing the average error in the task of placement in depth as a function of binocular disparity (expressed in $\mathrm{cm}$ ) and position

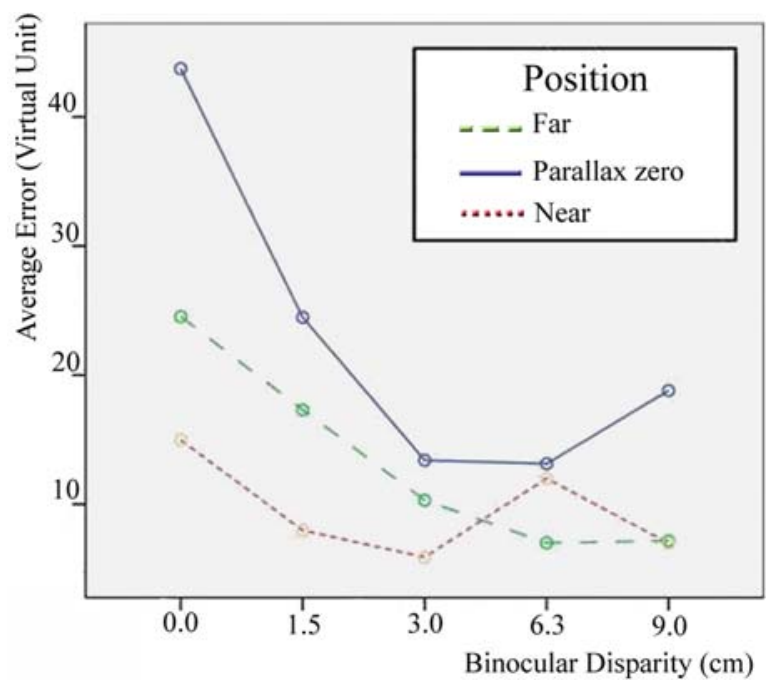

This figure will be printed in $\mathbf{b} / \mathbf{w}$

Results confirmed the high influence of binocular disparity cues. Regarding the influence of position, results logically revealed that the closer the virtual scene was located from the participant, the more effective was the placement. 
Moreover, averaged position errors always showed a positive sign; participants tended to place the torus closer than the cone, presenting a constant error skew.

Figure 2.4 also illustrates the influence of binocular disparity in depth perception. Results obtained in the case of position close to zero parallax plane showed an asymptotic reduction in the error when binocular disparity increased. Hence in this condition, for disparity values higher than $3.0 \mathrm{~cm}$ the error got stabilized. In the case of distant and near position, there were optimal values of binocular disparity (respectively $6.4 \mathrm{~cm}$ and $3.0 \mathrm{~cm}$ ). Therefore, a deterioration of depth perception was found when binocular disparity increased over this optimum.

However, in the case of near position, the error decreased for binocular disparity values higher than $6.4 \mathrm{~cm}$. The reason is that participants compensated an experimental misconception by bringing back dynamic objects. Effectively, regarding the proposed depth information, participants tended to place dynamic objects beyond the allowed manipulation limits.

\subsection{Discussion}

In the first experiment stage, results have demonstrated the high influence of the objects size in mechanisms of depth perception in contrast with binocular disparity. These findings are in accordance with previous works which have also analyzed this influence $[6,16]$. The analysis performed has revealed that objects size generates important errors in the perception of depth [16]. Thus, in the first stage of the experiment, participants' depth perception was built upon size cues and binocular cues neglected, due to the use of identically shaped objects.

Other experimental studies $[4,12,15]$ focused on analyzing the influence of several depth cues in depth perception mechanisms have also carried out experiments without avoiding possible size influences. This first study has outlined the necessity of removing size cues which can conceal other cues influence. Therefore, a new experimental design has been proposed for a second stage of the experiment. This design dealt with two differently shaped objects to avoid size cues predominance.

According to the aforementioned studies [4, 12, 15], the second study has been performed based on the assumption that depth perception is influenced by binocular disparities. Therefore, binocular cues should be an important factor to improve accuracy of tasks that basically consist in placing a virtual object in depth. Results of this second study have proved depth perception to be influenced not only by binocular disparity but also by objects position. Thus, participants' average error placing an object in depth has been influenced by both factors. Differences found in the magnitude of the average error as a function of binocular disparity among positions have suggested that, with moderate values of disparity, the closer the objects are, the more accurate the depth perception is. Nevertheless, this last relation has not been found in conditions of high binocular disparity. Therefore, high values of binocular disparity do not guarantee an accurate depth perception. This last finding supports the idea of using lower anti-natural binocular disparity even if the VE is strongly distorted $[4,15]$. 
The second experiment has also revealed that for objects placed close to the zero parallax plane, the depth perception error has tended asymptotically to a minimum value as the binocular disparity has been increased. This relation agrees with the results obtained by Rosenberg [4] in identical conditions. Nevertheless, Rosenberg has not considered the study of depth perception when objects were located further or closer than the zero parallax plane. In contrast with Rosenberg findings, in these last two cases, high values of binocular disparity have been found inefficient at improving accuracy of depth perception.

\subsection{Conclusion}

The understanding of depth perception mechanisms is a crucial issue to allow for accurate manipulation in a VE.

This work has evaluated the influence in depth perception of certain cues, such as size and binocular disparity. The size factor has been outlined as a possible cause of depth perception degradation. The findings presented substantiate that objects size strongly influences depth perception. Therefore, the analysis of the influence of other depth cues should be made avoiding this effect.

Previous studies have demonstrated binocular disparity as an important factor in depth perception. The analysis performed has also revealed that this influence is altered by the objects position. Thus, in case the virtual scene is located close to the zero parallax plane, an accurate perception of depth is ensured by a large range of binocular disparity values. Nevertheless, if the virtual scene is located much further or closer than the zero parallax plane, depth perception is accurate for binocular disparity included in a range slightly inferior to the natural eye separation.

Manipulating in depth in VEs is delicate and requires more investigation involving more participants. One possible approach of investigation relies on including proprioceptive and haptic cues to evaluate depth in a VE, by using haptic devices as manipulation interface.

Acknowledgments This study has been partially funded by the Spanish Ministry of Education and Sciences (Project TIN2006-15202-C03-02) and the council of Andalusia (Group PAI TIC171). Special thanks to Carmen Garcia Berdonés for her comments.

\section{References}

1. Alexander T et al. (2003) Depth perception and visual after-effects at stereoscopic workbench displays. Proc Virtual Real Annu Int Symp: 269-270

2. Bouguila L et al. (2000) Effect of Coupling Haptics and Stereopsis on Depth Perception in Virtual Environment. Proc. of the 1st Workshop on Haptic Human Computer Interaction: $54-62$

3. Kim WS et al. (1987) Visual Enhancements in pick and place tasks: Human operators controlling a simulated cylindrical manipulator. IEEE J Rob Autom, RA-3: 418-425

4. Rosenberg L (1993) Effect of interocular distance upon operator performance using stereoscopic displays to perform virtual depth tasks. IEEE Annu Virtual Reality Int Symp: 27-32 
5. Holliman N (2006) Three-Dimensional Display Systems. Handbook of Optoelectronics Vol II, Ed. Dakin JP and Brown RGW, Taylor \& Francis, Boca Raton

6. Wanger LR et al. (1992) Perceiving spatial relationships in computer-generated images. IEEE Comput Graphics Appl, 12: 44-58

7. Pfautz JD (2002) Depth Perception in Computer Graphics. University of Cambridge. PhD Thesis http://www.cl.cam.ac.uk/techreports/UCAM-CL-TR-546.pdf. Accessed September 2002

8. Stanney KM et al. (1998) Human factors issues in Virtual Environments: A review of the literature. Presence Teleoper. Virtual Environ, 7: 327-351

9. Bülthoff HH and Mallot HA (1998) Integration of depth modules: stereo and shading. J Opt Soc Am A 5: 1749-1758

10. Guibal CR and Dresp B (2004) Interaction of color and geometric cues in depth perception: when does "red" mean "near"? Psychol Res, 69:30-40

11. Sinai MJ et al. (1998) Terrain influences the accurate judgment of distance. Nature, 395: 497-500

12. Jones $\mathrm{G}$ et al (2001) Controlling perceived depth in stereoscopic images. Proc. SPIE Int Soc Opt Eng, 4297: 42-53

13. Hearn D and Baker MP (1995) Gráficas Por Computadora con OpenGL. Prentice Hall, Madrid

14. Lipton L (1991) Stereographics, Developers Handbook. StereoGraphics Corp

15. Holliman N (2004) A mapping perceived depth to regions of interest in stereoscopic images. Proc. SPIE Int Soc Opt Eng, 5291: 117-128

16. Sweet BT and Kaiser MK (2006) Integration of size and binocular disparity visual cues in manual depth-control tasks. Coll. Technic. Papers. AIAA Model. Simulat Technol Conf 2006, 2: $933-957$ 